\title{
ON COMPUTING THE INDEX IN THREE DIMENSIONS
}

\author{
K. R. MEYER ${ }^{1}$
}

Poincaré [1] gave a simple geometric procedure for computing the index of a critical point of a vector field in the plane. Let the trajectories induced by the field be tangent to a small circle about the critical point at a finite number of points. Let $e$ and $i$ denote the number of times the field is tangent to the circle from the outside and inside respectively. Then Poincare gave the following formula for the index

$$
I=1+(i-e) / 2 \text {. }
$$

A generalization of this formula to three dimensions will be discussed in this paper.

Now let $\nu$ be a smooth vector field defined in an open ball $V$ of $R^{3}$, where $V$ contains the unit 2 sphere $S^{2}$ in its interior. That is to say $\nu$ is a smooth map from $V$ into $R^{3}$. Furthermore assume that $\|\nu(x)\|=1$ for all $x \in S^{2}$ where \|\| is the Euclidean norm. Fix a coordinate system in $R^{3}$ and consider the 3 -frame $\Pi(x)$ at each $x \in V$ that is a parallel displacement of the original coordinate system. In this fixed coordinate system the point $(0,0,1)$ will be called the north pole and other geographic terminology consistent with this convention will be used.

The degree of the map $\nu \mid S^{2}: S^{2} \rightarrow S^{2}$ is called the index of the vector field $\nu$ with respect to $S^{2}$ and will be denoted by $I\left(\nu, S^{2}\right)$. In terms of the framing $\Pi$ the vector field $\nu$ has the form $\nu(x)=a_{1}(x) \Pi^{1}(x)$ $+a_{2}(x) \Pi^{2}(x)+a_{3}(x) \Pi^{3}(x)$ and so

$$
\nu \mid S^{2}: x \rightarrow\left(a_{1}(x), a_{2}(x), a_{3}(x)\right) .
$$

In the above $\Pi^{i}(x)$ denotes the unit vector in the $i$ th direction of the frame $I I$ at the point $x$.

A homotopy of the field $\nu$ will always be smooth and through fields $\nu_{t}$ such that $\left\|\nu_{t}(x)\right\|=1$ for all $x \in S^{2}$. A homotopy of the framing will always be smooth and through rigid rotations about each point. Both the above operations leave the index unchanged.

Let $\eta$ denote the unit outward normal vector field on $S^{2}$.

The following observation is due to M. M. Peixoto.

Lemma. There exists a smooth vector field $\mu$ that is $\epsilon$-homotopic to $\nu$ such that the field $\mu$ is tangent to $S^{2}$ only at a finite number of smooth

Received by the editors March 20, 1967.

1 This research was supported by the National Aeronautics and Space Administration under Contract No. NAS8-11264. 
circles $C_{1}, \cdots, C_{p}$. These circles are the boundaries of a finite number of smooth connected 2 submanifolds with boundary $A_{1}, \cdots, A_{q}$ such that the field $\mu$ has a positive component in the direction.of $\eta$ at each interior point of $A_{i}, i=1, \cdots, q$. At each point of $B=S^{2}-\bigcup_{i=1}^{q} A_{i}$ the field $\mu$ has a negative component in the direction of $\eta$.

Proof. Consider the map $g: S^{2} \rightarrow R$ defined by taking the component of $\nu$ in the direction of $\eta$. By applying Sard's Theorem [2] in the usual way one constructs $\mu$ from $\nu$.

Henceforth it will be assumed that the vector field has been prepared in accordance with the above lemma. The $C_{i}, i=1, \cdots, p$ will be called circles of contact and any region where the field has a positive (negative) component in the direction of $\eta$ will be called a region of egress (ingress). Thus the interior of $A_{i}, i=1, \cdots, q$, is a region of egress and $B$ is a region of ingress.

Let the $A_{i}, i=1, \cdots, q$ be oriented as submanifolds of $S^{2}$ and the $C_{i}, i=1, \cdots, p$ be oriented as boundaries of the appropriate $A_{j}$.

There exists a framing $\Sigma_{j}$ of $V$ that is smoothly homotopic to $\Pi$ such that along $C_{j}$ the last component of $\Sigma_{j}$ is $\eta$. The field $\mu$ on $C_{j}$ can be expressed as $b_{1}(x) \Sigma_{j}^{1}(x)+b_{2}(x) \Sigma_{j}^{2}(x)$. The degree of the map $h: C_{j} \rightarrow S^{1}$ defined by $h: x \rightarrow\left(b_{1}(x), b_{2}(x)\right)$ will be called the index of $\mu$ with respect to $C_{j}$ and will be denoted by $I\left(\mu, C_{j}\right)$. Clearly this index does not depend on the choice of $\Sigma_{j}$.

We can now state our main result.

Proposition. The index of the field such that all of $S^{2}$ is a region of egress is +1 . In all other cases

$$
I\left(\mu, S^{2}\right)=-1+\sum_{j=1}^{p} I\left(\mu, C_{j}\right) .
$$

Proof. Clearly the theorem holds if all of $S^{2}$ is a region of egress so we can assume that not all of $S^{2}$ is a region of egress. The field $\mu$ can be deformed to the field $\mu^{\prime}$ so that all the circles of contact of $\mu^{\prime}$ lie north of the Tropic of Capricorn and so that the region south of the Tropic of Capricorn is a region of ingress. Moreover the deformation can be constructed so that there is a one to one correspondence between the circles of contact of $\mu$ and $\mu^{\prime}$ and such that the corresponding indices are the same. The frame $\Pi$ can be deformed to a frame $\Sigma$ where $\Sigma$ has the following properties (i) north of the Tropic of Capricorn the last component of $\Sigma$ is $\eta$, (ii) in the southern hemisphere $\Pi$ and $\Sigma$ agree, and (iii) between the equator and the Tropic of Capricorn the homotopy between $\Sigma$ and $\Pi$ can be accomplished by a rotation through an angle less than or equal to $3 \pi / 8$. 
Now $\mu^{\prime}(x)=b_{1}(x) \Sigma_{1}(x)+\cdots+b_{3}(x) \Sigma_{3}(x)$. The degree of the map $w: S^{2} \rightarrow S^{2}$ given by $w: x \rightarrow\left(b_{1}(x), \cdots, b_{3}(x)\right)$ is $I\left(\mu, S^{2}\right)$. Now $w$ maps the circles of contact into the equator and the regions of egress into the northern hemisphere. Now we count the number of times the northern hemisphere is covered.

Let $F=C_{1} \cup \ldots \cup C_{l_{1}}$ be the boundary of $A_{1}, S^{1}$ the equator of $S^{2}$ and $N$ the northern hemisphere of $S^{2}$. From the following commutive diagram

$$
\begin{aligned}
& H_{2}\left(A_{1}, F\right) \stackrel{\partial}{\rightarrow} H_{1}(F) \\
& \downarrow w_{*} \quad \downarrow w_{*} \\
& H_{2}\left(N, S^{1}\right) \stackrel{\partial}{\leftrightarrow} H_{1}\left(S^{1}\right),
\end{aligned}
$$

where $H_{i}(X, Y)$ is the $i$ th in teger homology group of $X$ modulo $Y$ and $w_{*}$ is the homomorphism induced by $w$, it follows that if $I\left(\mu^{\prime}, C_{i}\right)=k_{i}$, that is the generator of $H_{1}\left(C_{i}\right)$ is mapped by $w_{*}$ onto $k_{i}$ times the generator of $H_{1}\left(S^{1}\right)$, then the generator of $H_{2}\left(A_{1}, F\right)$ is mapped by $w_{*}$ onto $\left(k_{1}+\cdots+k_{l_{1}}\right)$ times the generator of $H_{2}\left(N, S^{1}\right)$. In the above "the generator" is to be taken as the generator corresponding to the oriented manifold itself.

Now if $\mu_{1}$ is the field, defined on $S^{2}$, obtained from $\mu^{\prime}$ by changing the sign of the last component of $\mu^{\prime}$ in the region $A_{1}$ then by the above

$$
I\left(\mu^{\prime}, S^{2}\right)=I\left(\mu_{1}, S^{2}\right)+\sum_{i=1}^{l_{1}} I\left(\mu^{\prime}, C_{i}\right) .
$$

By repeating this process for each region of egress the theorem follows since in the last step

$$
I\left(\mu^{\prime}, S^{2}\right)=I\left(\mu_{q}, S^{2}\right)+\sum_{i=1}^{q} I\left(\mu^{\prime}, C_{i}\right)
$$

and where $\mu_{q}$ is a field such that all of $S^{2}$ is a region of ingress and so $I\left(\mu_{q}, S^{2}\right)=-1$.

REMARK 1. This formula yields an effective geometric procedure for computing the index of a critical point in three dimensions. The formula shows that computing the index in three dimensions can be reduced to computing the index along several circles. The Poincaré formula can be used to compute these indices provided one is careful about orientations.

REMARK 2. The formal part of the proof given above goes over with only minor changes for any $n \geqq 2$. For $n=2$ one obtains Poincaré's formula in a slightly different form. For $n>3$ the circles of contact 
could be quite arbitrary differential manifolds and so the formula says that the index in $n$-dimensions can be computed by computing the degree of a map from an $n-2$ manifold in to the $n-2$ sphere. The above procedure does not seem to indicate how to compute the degree of this map and so the inductive procedure stops. Thus the above is only effective in dimensions 2 and 3.

\section{REFERENCES}

1. H. Poincaré, Sur les courbes définies par une équation differentielle, Oeuvres, Vol. 1, Paris, 1892.

2. S. Sternberg, Lectures on differential geometry, Prentice-Hall, Englewood Cliffs, N. J., 1962.

BROWN UNIVERSITY 